Téoros

Revue de recherche en tourisme

\title{
La spécificité des paysages naturels de Charlevoix
}

\section{Jean Raveneau}

Volume 17, numéro 1, printemps 1998

Le tourisme dans un Pays à part : Charlevoix

URI : https://id.erudit.org/iderudit/1072370ar

DOI : https://doi.org/10.7202/1072370ar

Aller au sommaire du numéro

Éditeur(s)

Université du Québec à Montréal

ISSN

0712-8657 (imprimé)

1923-2705 (numérique)

Découvrir la revue

Citer cet article

Raveneau, J. (1998). La spécificité des paysages naturels de Charlevoix. Téoros, 17(1), 9-14. https://doi.org/10.7202/1072370ar d'utilisation que vous pouvez consulter en ligne.

https://apropos.erudit.org/fr/usagers/politique-dutilisation/ 


\section{tais.r.}

\section{LA SPÉCIFICITÉ DES PAYSAGES}

\section{naturels de Charlevoix}

Jean Raveneau, professeur Département de géographie, Université Laval

Ce texte resume les grands traits et la genèse des paysages naturels de Charlevoux. Ceuxci sont caractérisés par un relié étagé é comparimenté, avec de fortes dénivellations. De hauts plateaux et des valles profondes occupent l'arriere-pays alors que mer et montagne se rejoignent sur le littoral du Saint-Laurent. Les payages de Charlevoix refletent differents types de contacts, gelogiques et biogeggraphiques entre autres, Quelques moyens de decouverte visuelle de ces paysages sont esquissés en fin d'article.

L'observateur qui découvre Charlevoix pour la première fois est frappé par l'ampleur du relief qui s'offre à ses yeux et par la rencontre harmonieuse des paysages de mer, de plateaux, de montagnes et de vallees. Son regard peut suivre les grands abrupts plongeant directement dans l'estuaire du Saint-Laurent, remonter l'une ou l'autre des deux grandes vallées qui partent du fleuve, pour s'élever sur les plateaux jusqu' aux sommets culminant à plus de 1100 mètres à une vingtaine de kilomètres seulement à l'intérieur des terres. L'impression générale qui se dégage est celle d'un paysage montueux, montagneux par endroits, impression accentube par la couverture neigeuse en hiver et au printemps, de même que par la présence de vastes forêts de coniferes ou d'essences mélangées sur' les plateatux. sur les versants et dans les vallées. En contrepartie de l'onniprésence des plateaux et des montagnes s'impose l'ampleur du fleuve Salint-Laurent, qui s'est dilaté brusquement à la pointe de l'île d'Orléans pour devenir un estuaire large de 15 à 20 kilonètres, composante indissociable des paysages de Charlevoix et de l'histoire du peuplement de la région. Dans les lignes qui suivent nous nous proposons de résumer les traits essentiels et la spécificité des paysages naturels de Charlevoix', en mettant en évidence les caractéristiques qui ont justifié l'octroi à la région du statut de Réserve mondiale de la biosphère.

\section{UNE HISTOIRE GÉOLOGIQUE MOUVEMENTÉE}

On ne peut comprendre les paysages de Charlevoix sans faire référence aux grands ensembles physiographiques et à des événements marquants de l'histoire géologique de la région, que nous résumerons de manière très schématique. Le socle de Charlevoix appartient au Bouclier canadien, formé de roches précambriennes très dures, vieilles de plus de 900 millions d'années. Cette partie du Bonclier se situe dans la province géologique de Grenville et dans la section orientale de la région physiographique des Laurentides. Elle comprend principalement les roches des séries gabbro-anorthositique (massif d'anorthosite de Saint-Urbain), charnockitique (la plus étendue), des roches migmatiques enclavées, des paragneiss, des gneiss et des granites. Au moment de la surrection du massif des Laurentides, il y a 700 millions d'années, un fossé d'ef- fondrement, un rift, s'est forme sur la marge sud-est du Bouclier, le long d'un système de failles orientes nord-est-sudouest. Ce fossé a ta été envahi par un océan au fond duquel se sont formés les dépôts qui allaient par la suite constituer les formations appalachiennes. Durant la période ordovicienne (de 510 à $440 \mathrm{mil}$ lions d'années), la mer a envahi progressivement le Bouclier et des sédiments marins se sont accumulés â la surface sur une épaisseur de 200 mètres. Vers 450 millions d antees, les fonds marins se sont soulevés au sud-est du Bouclier, donnant naissance aux Appalaches. Ce soulèvement provoqua, au contact du Bouclier, une immense cassure encore bien visible aujourd hui, la faille du Sant-Laurent, qui suit le littoral de Cap-Tourmente ả SaintSiméon. Il s sensuivit l'effondrement du bassin de Québee (plate-forme du SaintLaurent), entre le Bouclier et les Appalaches, dans lequel s'installera plus tard le lit du fleuve Saint-Laurent (Rondot. 1979). Ceci explique que l'on rencontre aujourd'hui, en contrebas de la cote de Charlevoix, les vestiges de dépôts ordoviciens (grès, calcaires, dolomies) qui se trouvaient originellement à la surface du Bouclier. Ces formations peuvent être observées à Cap-à-I+Aigle, Saint-Irénée et Cap-aux-Oies.

Un autre temoin du soulevement des Appalaches est l'île aux Coudres, qui a émergé à cette époque. Elle est séparée du Bouclier par la cassure de la ligne de Logan qui court au fond du fleuve parallèlement à la faille du Saint-Laturent, à deux kilomètres au sud-est de celle-ci environ. La ligne de Logan marque le contact entre le Bouclier et les formations appalachiennes, auxquelles les roches de l'île aux Coudres appartiennent. L'île aux 
Coudres est la troisième plus grande île de l'estuaire du Saint-Laurent, apres l'île d'Anticosti et l'île d'Orléans.

Au Dévonien, il y a 360 millions d'années, survient un évênement majeur qui va façonner de manière indélébile le paysage de Charlevoix: il s'agit de l'impact d'une météorite géante, un astroblène ${ }^{2}$, à l'emplacement actuel du mont des Éboulements. Cette météorite de deux kilomètres de diametre et pesant 14 milliards de tonnes a frappé le bord du Bouclier à une vitesse de 10 à 20 kilometres/seconde (Rondot 1979 et 1995). Elle a d'abord creusé un cratère de 4 à 5 kilomètres de profondeur et de 28 kilomètres de diamètre, puis un réajustement de la croûte terrestre s'est produit, entraînant une remonteve verticale de plus de quatre kilomètres au centre du cratère, et formant un pointement dont le mont des Éboulements est le vestige. Ce relèvement consécutif à l'impact météoritique a provoqué à son tour un effondrement circulaire de 56 kilomètres de diamètre, balisé par une série de lignes de faille, occupé aujourd hui par la dépression annulaire où coulent les rivières du Gouffre et Malbaie. Le flanc externe de cette dépression est bordé par un escarpement qui se dresse au contact du rebord du haut plateau des Laurentides et des gradins du versant nord-ouest de la vallée de la rivière du Gouffre, entre Saint-Urbain et NotreDame-des-Monts, On suit également la trace de l'effondrement annulaire sur la rive sud du fleuve, dans la forme arquée du rivage vis-ă-vis de La Pocatière.

Les bombements, les soulèvements, les effondrements et l'impact météoritique qui ont déformé la croûte terrestre de la région de Charlevoix au cours de l'histoire géologique ont engendré de nombreuses lignes de cassure ou failles. Des ajustements continus se sont poursuivis jusqu'à nos jours le long de ces failles, en particulier dans la zone de faiblesse qui suit le Saint-Laurent dans l'axe de la ligne de Logan, engendrant une activité séismique qui se manifeste de temps à autre par des tremblements de terre d'intensité variable. Le séisme le plus considérable rapporté durant la période historique fut celui de 1663; il provoqua un énorme glissement de terrain sur les débris duquel a êté construit Saint-Joseph-de-la-Rive, au pied du village des Éboulements.

Le dernier million d' années de l'histoire géologique (ère quaternaire) a été marqué par les avancées et retraits successifs de plusieurs glaciations, dont la plus récente prit fin ill y a 9 à 10000 ans (glaciation wisconsinienne). Ces glaciers ont été de puissants agents d'érosion, sculptant, rabotant et striant la surface du socle précambrien, façonnant des drumlins (au nord de Saint-Hilarion), des roches moutonnées (affleurements rocheux polis en forme de dos de baleine), surcreusant des vallées préglaciaires en leur donnant une forme en $\mathbb{U}$. Quelques-unes de ces vallées peuvent être observées dans le parc des Hautes-Gorges-de-la-rivière-Malbaie. Certaines d'entre elles se referment en amphitheâtre, formant des cirques spectaculaires, notamment au lac du Tonnerre, au lac Prime et au lac Noir. En fondant les glaciers ont laissé différents types de dépôts. D'abord la moraine de fond (till). omniprésente, dont la coloration jaune est typique des sols du plateau intermédiaire de Charlevoix. Des cordons morainiques et des eskers forment des alignements de buttes, notamment dans la région de SaintAimé-des-Lacs et sur le plateau au-dessus de Saint-Irénée. Des terrasses et des deltas fluvioglaciaires peuvent être observés sur les flanes de la partie supérieure des vallées des rivieres du Gouffre et Malbaie, alors que des épandages fluvioglaciaires se sont répandus au fond des mêmes vallées. Les eaux de fonte de la dernière glaciation ont donné naissance à la mer de Champlain, qui a laissé des dépôts argileux jusqu à une altitude de 180 mètres dans les vallées, tout en remaniant les dépôts fluvioglaciaires antérieurs. Au fur et à mesure que le continent se relevait et que l'eau se retirait, des terrasses se formaient sur les versants des vallées, terrasses qui sont aujourd'hui ravinées par l'érosion.

\section{UN RELIEF AMPLE, ÉTAGÉ ET COMPARTIMENTÉ}

Les événements géologiques que nous venons de décrire sommairement ont contribué à façonner les traits généraux du relief de Charlevoix tels que nous les connaissons aujourd' hui. Ces traits peuvent être schematisés à 1'aide de trois termes: étagement, compartimentation et ampleur des dénivellations. En altitude, on peut distinguer trois niveaux à partir du fleuve Saint-Laurent. Le premier niveau est celui de l'étage inférieur du littoral, de l'ille aux Coudres et de la partie inférieure des deux grandes vallées (rivière du Gouffre et rivière Malbaie) situés à quelques dizaines de mètres au-dessus du niveau de l'estuaire de Saint-Laurent. Le deuxième niveau est constitué par un platedu intermédiaire, mollement ondulé, d'une altitude de 300 à 500 mètres, enserré entre les vallées des rivières du Gouffre et Malbaie et dont le village de SaintHilarion occupe approximativement le centre; il est dominé par le massif des Eboulements, qui culmine à 768 mètres. Il se prolonge au nord-est en s'élevant jusqu'à 600-700 mètres, puis en s"abaissant jusqu'au Saguenay. Au sud-ouest le plateau intermediaire s' etire vers SaintPlacide et Saint-Tite-des-Caps à des altitudes de 450 à 760 mètres. Le plateau supérieur constitue le troisième niveau, à l'ouest et au nord-ouest de la région, à des altitudes comprises entre 600 et 1000 mètres avec des sommets culminant à plus de 1100 mètres.

Le plateau supérieur correspond à la surface de la vieille pénéplaine précambrienne du massif des Laurentides, dont une partie est occupée par la Réserve faunique des Laurentides. Cette surface peut se subdiviser en deux parties bien différenciées: 1) au nord-est de la rivière Malbaie, le plateau ne dépasse pas 900 mètres et s'abaisse progressivement vers le Saguenay. Il est parcouru par une série de vallées très évasées, colmatées par divers dépôts glaciaires; 2) à l'ouest d'une ligne joignant le mont Elie à Saint-Placide, I'altitude de la surface du haut plateau varie de 600 à 1000 mètres, présentant une topographie tres accidentée, marquée par une sếrie de fractures orthogonales, de nombreuses vallées en U d'origine glaciaire, des vallées suspendues, des cirques glaciaires, etc. Les sommets les plus élevés se retrouvent à l'est du canyon de la rivière Malbaie (mont des Érables, mont Élie, mont Jêrémie, culminant à environ 1020 à 1050 mètres), au nord de Saint-Urbain (mont du Lac des Cygnes, mont du Lac à I'Empêche, 945 et 914 mètres) et à l'ouest de la rivière Sainte-Anne dụ Nord. A la surface de ce haut plateau. les lignes de fracture entrecroisées - particulièrement visibles sur les vues aériennes et satellitaires - constituent autant de lignes de faiblesse dans la roche en place qui ont été exploitées par les agents d'érosion, dont le travail des cours d'eau, tout au long de l'histoire géologique. Ces fractures entrecroisées dégagées par l'êrosion et surcreusées par les glaciers, expliquent notamment le tracé en baionnette (ou en équerre) et la formation du canyon de la rivière Malbaie dans la section des Hautes-Gorges, de même que le profond encaissement de la rivière Sainte-Anne du Nord. 
La compartimentation du relief de Charlevoix est déterminée par la présence de vallées fortement déprimées et d'abrupts marqués. Ainsi, les deux vallées de la rivière du Gouffre et de la rivière Malbaie forment un arc enserrant le plateau de Saint-Hilarion et débouchent dans le SaintLaurent à 300 metres au-dessous de la surface du plateau intermédiaire. Sur le haut plateat on a dêjă mentionné que la rivière Sainte-Anne du Nord et la haute rivière Malbaie coulent à l'intérieur de véritables canyons: le lit de la première est situé à plus de 450 mètres au-dessous de la surface du plateau, alors que celui de la seconde est dominé par des falaises de 750 mètres dans le parc des HautesGorges, avec des parois verticales pouvant atteindre 300 metres. Dans les deux cas, l'écartement de la partie supérieure des flancs de la vallée ne dépasse pas 1500 mètres.

Un autre élement de la compartimentation topographique de là région est déterminé par les escarpements correspondant à la tombée du plateau supérieur sur le plateau intermédiaire, sur le pourtour de la dépression annulaire drainée par les rivières du Gouffre et Malbaie. Cet escarpement. atteignant par endroits une dénivellation de 600 metres, correspond au kmur d'enceintes de la dépression externe de l'astroblème de Charlevoix (Rondot, 1979 et 1995). Il donne la fausse impression d'une chaîne de sommets quand on le voit du sud; en réalité, les sommets qui le dominent (mont Blanc, mont du Lac des Cygnes, mont du Lac à l'Empêche, mont Élie, etc.) sont situés à peu près à la même altitude que la partie la plus haute du plateau de la Réserve faunique des Laurentides, située au nord-ouest de la région. Pour un observateur parcourant le rang Saint-Jean-Baptiste, entre Saint-Urbain et Notre-Dame-des-Monts, cet escarpement en are de cercle ferme l'horizon a l'ouest et au nord-ouest et constitue une barrière visuelle marquant la limite d'un autre monde.

Au dernier palier de l'étagement, le pláteau intermediaire tombe abruptement sur le littoral du Saint-Laurent. Au cap Maillard, à Petite-Rivière-Saint-François, la dénivellation est de 750 mètres sur une distance horizontale de moins de 1500 mètres. Cette dénivellation spectaculaire a été mise à profit pour le tracé des pistes de la station de ski Le Massif, du haut desquelles les skieurs peuvent contempler le panorama grandiose du fleuve, du littoral, de l'île aux Coudres et des Appa-

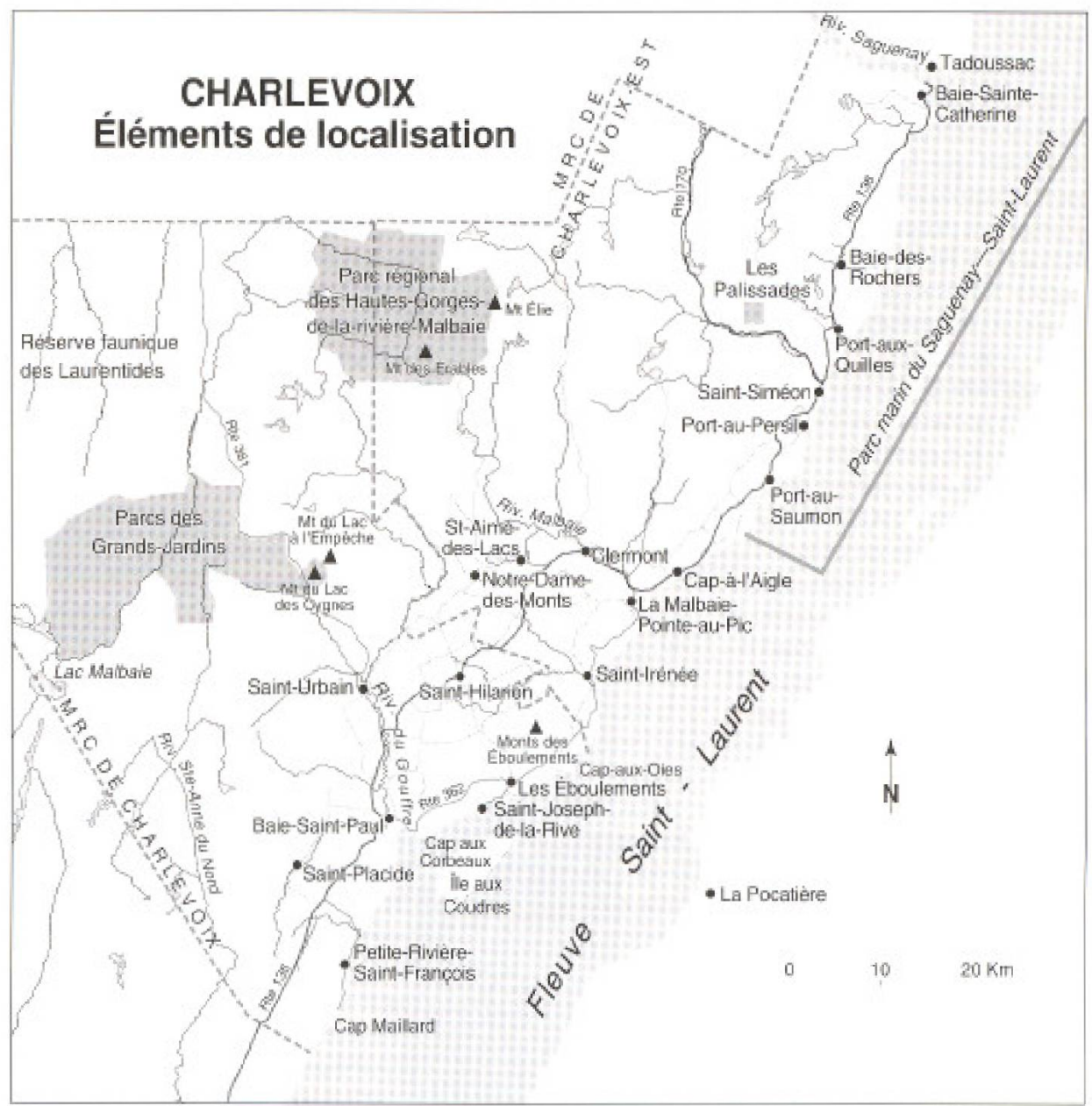

laches, au-delà de la rive sud du SaintLaurent. Entre le cap aux Corbeaux et Pointe-au-Pic le littoral affecte un tracé en forme d'arc, avec un bombement pointant dans le Saint-Laurent à Cap-aux-Oies, epousant les contours du mont des Éboulements et de l'astroblème. La dénivellation se maintient à 300 mètres du cap aux Corbeaux aux Eboulements, alors qu'elle s'abaisse vers le nord-est, pour descendre à 150-180 mètres aux environs de Saint-Irénée et Pointe-au-Pic. La dépression de la rivière Jean-Noël, aboutissant dans le fleuve au village de Saint-Irénée, constitue la seule échancrure importante du littoral en dehors des deux vallees principales. De Cap-à-l'Aigle jusqu'au Saguenay, le littoral s'élève à nouveau jusqu" 300 mètres au-dessus du fleuve. Son tracé est caractérisé par une série d'indentations et de caps entre lesquels $s$ insêrent des anses et des baies fortement encaissées, très pittoresques malgré leur faible amplitude. Dans ces havres se sont installées de petites communautés humaines, vivant autrefois des ressources du fleuve: Port-au-Saumon. Port-au-Persil. Port-aux-Quilles, Baie-des-Rochers.

\section{AU CONTACT DU MONDE MARIN}

Au pied du littoral, au contact de la terre et du fleuve, se couvrant et se découvrant au rythme des deux marées journalières (celles-ci ont une amplitude de 4 à 5 mètres), l'estran constitue une composante indissociable du paysage littorall. S'étendant sur une largeur variable, de 100 à 600 mètres selon les endroits, il prend divers aspects: celui d'une plate-forme d'abrasion rocheuse parsemée de blocs glaciels, comme à Petite-Rivière-Saint-François, Cap-aux-Oies, Cap-à-l'Aigle; ou d'un estran vaseux près de Baie-Saint-Paul, à Saint-Joseph-de-la-Rive et à Pointe-auPic-La Malbaie. L'estran vaseux abrite par endroits des marais salés à Spartine, où poussent les afoins saléss. (Spartines a fleurs alternes), formant de riches prairies marines autrefois exploitées par les habitants riverains, notamment ă 1 thle aux Coudres où une baie porte le nom de baie de la Prairie. A l'embouchure des vallées du Gouffre et de la rivière Malbaie, l'estran est recouvert par les sables deltaïques des deux rivières. A Baie-Saint-Paul, un 
cordon littorạl protège un marais, entre l'estran et la plaine alluviale. En aval de Cap-à-l'Aigle l'estran disparait presque complètement. sauf dans la baie des Rochers qui forme un bel amphithéâtre, puis répparaît à Baie-Sainte-Catherine. Plusieurs plages de sable fin émaillent le littoral, à Baie-Saint-Paul, Saint-Irénée, Saint-Siméon et Baic-Sainte-Catherine, invitant le visiteur a la détente.

La section du Saint-Laurent située entre Cap-Tourmente et l'embouchure du Saguenay est qualifiée de moyen estuaire. entre l'estuaire fluvial qui se termine à l'île d'Orléans et l'estuaire maritime qui commence à Tadoussac. Dans cette section du moyen estuaire l"eau douce fait rapidement place à l'cau salée: la salinité de l'eau, qui est de $\|$ (parties pour mille) à Cap-Tourmente, passe à $10 \%$ â PetiteRivière-Saint-François puis à $20 \%$ à Capaux-Oies. Entre l'île d'Orléans et l'île aux Coudres, le mélange des eaux douces et des eaux salées forme une zone de turbidité (le bouchon vaseux) en raison du brassage des matières en suspension dans $I^{+}$eau, qui sont piégées par le renversement des courants de marée. Au confluent du fjord du Saguenay, les eaux froides des profondeurs du Saint-Laurent remontent en surface en se butant au seuil sous-marin qui ferme le fjord et entrent en contact avec les eaux plus chaudes de la surface, créant une zone d'intense activité biologique. Cette zone riche en nourriture attire une faune marine exceptionnelle, dont les bélugas et les baleines sont les représentants les plus médiatisés auprès du public. C'est pour protéger, conserver et mettre en valeur cette aire marine remarquable qu'a été créé le parc marin SaguenaySaint-Laurent, qui offre au public de multiples possibilités de découvrir la faune, la flore et les paysages du milieu environnant.

\section{DE LACS EN RIVIĖRES}

La masse imposante du Saint-Laurent ne doit pas faire oublier la richesse du réseau hydrographique qui draine plateaux el vallées a l'intérieur de la région. Le haut plateau des Laurentides est un véritable château d'eau qui reçoit $1500 \mathrm{~mm}$ de pluie par an en moyenne. La nature des roches du substrat, I'action des glaciers et les fortes précipitations expliquent la présence de plus de 1000 lacs dans la région, dont treize ont une étendue de plus de 100 hectares. Les plus grands sont les lacs Pikauba, des Martres, Malbaie, à Jack. La rivière Malbaie prend sa source dans les deux derniers lacs et la rivière du Gouffre dans le lac des Coeurs. Les bassins de ces deux rivières couvrent respectivement $1850 \mathrm{~km}^{2}$ et $1000 \mathrm{~km}^{2}$. Les autres bassins de drainage sont beaucoup plus réduits mais les petits cours d eau qui dévalent les pentes et escarpements n'en sont pas moins pittoresques. De nombreuses chutes et cascades ponctuent en effet le cours des rivières et ruisseaux sur la bordure des plateaux et les versants des vallées. On peut citer entre autres la chute des Martres (200 metres) dans les Hautes-Gorges de la rivière Malbaie, celles de la rivière Comporté (chutes Fraser) au nord-est de La Malbaie, la chute de la rivière du Moulin a Baie-Saint-Paul, etc. Tous ces lacs, rivières et ruisseaux se prêtent à des activités sportives et récréatives variées.

\section{LES PAYSAGES VÉGÉTAUX: DE L'ÉRABLIĖRE À LA TOUNDRA}

On ne peut présenter les paysages de Charlevoix sans évoquer le couvert végétal qui revêt les différents niveaux et compartiments topographiques décrits plus haut. Les caractéristiques de lă végétation traduisent de multiples influences topoclimatiques: situation en latitude. étagement en altitude, orientation des versants, nature et épaisseur des sols, conditions du drainage, caractéristiques du climat régional et local, etc. La végétation de Charlevoix reflète une situation de contact à la limite nord de la forêt feuillue et à la limite sud de la forêt borḱale coniferrienne. La zone de la forêt feuillue correspond au domaine de l'érablière à bouleau jaune et du hêtre. Elle est confinee à quelques aires limitées au premier niveau topographique entre 0 et 300 mètres, dans la partie inférieure des deux vallées principales, sur I'ile aux Coudres et au bas de l'escarpement littoral à Petite-Rivière-Saint-François où l'on peut observer de belles érablières. La forêt mixte, domaine de la sapinière à bouleau jaune et de la sapinière à érable rouge couvre le plateau intermédiaire central et son prolongement vers le nord-est: la sapinière à bouleau jaune occupe aussi les gradins en forme d'anneau sur la bordure externe de l'are des vallées de la rivière du Gouffre et de la rivière Malbaie. Au-dessus de 600 mètres le haut plateau est occupế par la forêt coniférienne. En åltitude se succèdẹt le domaine de la sapiniere à bouleau blanc, celui de la sapinière à épinette noire. puis de la pessière noire. Sur les sommets règnent des conditions climatiques extrềmes où ne peuvent subsister que les arbres rabou- gris de la pessière à Kalmia et Cladonie. Sur le haut des cimes battues par les vents, entre 950 et 1100 mètres, seule une végétation de toundra - maigres arbustes, mousses et lichens -, peut résister aux rigueurs du climat. Ce millieu ầ caractère arctique est fréquentế par un troupeau de caribous dans le Pare des Grands-Jardins.

Le contact entre les differents types de peuplements, loin d'être linéaire, epouse les particularités des sites topographiques contrastés, et provoque des juxtapositions de formations très diversifiés dans lesquelles feuillus et coniferes alternent sur de courtes distances. L'effet visuel de cette alternance est particulièrement saisissant en automne et au printemps. L'étagement et les juxtapositions de differentes formations végétales peuvent être observés localement dans les vallées encaissées comme celle de la rivière Malbaie, dans le secteur des Hautes-Gorges, où l'on voit se succéder, du bas vers le haut, les ormes et les érables, les bouleaux blancs et les sapins, puis la pessière noire et l'étage alpin de la toundra au sommet du plateau a 800 metres au-dessus de la riviere.

La forêt de Charlevoix est essentiellement une forêt secondaire, résultant d'une regénération après des feux ou des coupes répétées. Elle a aussi été largement deffrichéc pour l'agriculture dans les deux vallées et sur le plateau intermediaire. Après avoir atteint une extension maximum au milieu du $X X{ }^{\circ}$ siecle, les défrichements agricoles régressent, surtout sur le plateau intermédiaire où ne subsiste qu'une agriculture marginale. Les espaces agricoles contribuent pourtant au charme et a la diversité des paysages de Charlevoix. Leur recul devant la forêt résulte en une monotonie des formes et des couleurs qui amene un appatuvrissement de l"attrait visuel des espaces abandonnés.

Le couvert végétal de Charlevoix reflète en partic les oppositions climatiques entre la mer et la montagne. Le littoral du SaintLaurent bénéficie de températures moyennes plus clémentes de deux à trois degrés qu'à la surface des plateaux et la saison sans gel y est plus longue; les précipitations y sont aussi deux fois moins élevées. La partie supérieure des vallées (vers Saint-Urbain notamment) semble jouir de conditions d'ensoleillement plus favorables que sur la côte, avec un taux d'humidité moins élevé. Sur le littoral l'état du ciel est plus variable. En hiver règnent de fréquents brouillards dus à la difference de température entre l'euu froide du fleuve 
et l'air ambiant plus chaud. Cela engendre des conditions de lumière changeantes que de nombreux peintres ont su exprimer sur leurs toiles.

\section{LA DÉCOUVERTE VISUELLE DE CHARLEVOIX}

De nombreuses possibilités existent pour découvrir visuellement les paysages de Charlevoix, que ce soit par terre, par mer ou même du haut des airs. Toutefois, pour la majorité des visiteurs, c'est en suivant les routes, les chemins ou les sentiers de randonnée que l'on peut accéder à des points de vue panoramiques offrant la contemplation de paysages variés sur des distances plus ou moins grandes. Ce sont les sections d'itinéraires routiers en surplomb, situées en bordure du plateau intermédiaire, au contact des deux grandes vallées et du littoral, qui offrent les meilleures possibilités de découverte visuelle des paysages. Les itinéraires en surplomb coincidant avec les paysages les plus spectaculaires, sont situés sur le versant ouest de la vallée du Gouffre (descente de la route 138 vers Baie-Saint-Paul), au nord et à l'ouest du plateau de Saint-Hilarion (rang Saint-Jean-Baptiste, entre SaintUrbain et Notre-Dame-des-Monts), sur les hauteurs du littoral (route 362 ) aux allentours de Cap-aux-Corbeaux, des Éboulements, de Cap-a-l'Aigle, et sur la route ceinturant l'île aux Coudres. Les itinéraires situés à la surface du plateau de SaintHilarion et sur le littoral en aval des Éboulements offrent une vue généralement moins étendue, mais néanmoins empreinte de variété, traversant des zones de transition, des seuils, entre des types de paysages différents. Aux itinéraires en surplomb succedent des itincraires encaissés, en dépression, où l'oeil est sollicité vers les hauts des versants. On les trouve dans les deux vallées principales, mais aussi lorsque la route longe l'estran, au pied du littoral, à Petite-Rivière-SaintFrancois ou à Saint-Irénée. Sur le plateau intermédiaire, les routes traversant des paysages de hautes collines suivent également des itineraires encaissés. C'est dans les Hautes-Gorges-de-la-rivièreMalbaie que I'encaissement du sentier offre les sensations les plus spectaculaires.

En suivant les routes et les sentiers on accède à des points de vue panoramiques dont les plus spectaculaires ont fait l'objet d'aménagements (belvédères). Les points de vue que l'on peut qualifier de srégionaux $\%$ offrent des panoramas portant a plusieurs dizaines de kilomètres et permet- tent d'observer la majorité des grands ensembles de paysages de Charlevoix, soit le Saint-Laurent (et au-delà, la rive sud), le littoral, 1'ûle aux Coudres, les vallées du Gouffre et de la rivière Malbaie, le plateau de Saint-Hilarion, le plateau intermédiaire périphérique, lâ bordure du haut plateau des Laurentides et son piémont et le haut plateau Jui-même. Mentionnons I'emplacement de quelques-uns de ces points de vue majeurs: le belvédère de la côte Saint-Antoine (route 138) en descendant vers Baie-Saint-Paul, le village des Éboulements at le haut de la côte qui descend vers Saint-Joseph-de-laRive, la pointe de l'Islet et la pointe du Bout d'en Bas à l'île aux Coudres, le haut du rang Sainte-Mathilde à Cap-à-l'Aigle, la croix de Clermont, l'eglise de SaintHilarion (le vieux cimetière), le sommet du mont du Lac des Cygnes, dans le parc des Grands-Jardins; le sommet du mont des Éboulements offre également une vue circulaire remarquable, mais il est peu accessible. Il existe bien sûr beaucoup d'autres points de vue d'étendue plus limitee, le long des routes 138 et 362 , mais aussi sur le circuit de l'arrière-pays, entre Saint-Urbain et Saint-Aimé-des-Lacs. en suivant le chemin du Pied des Monts ou en montant vers le parc des HautesGorges-de-la-rivière-Malbaie. Il ne faut pas hésiter non plus à s'écarter des routes principales, sur des chemins de gravier, pour se laisser surprendre par des points de vue inattendus.

Pour les visiteurs désireux d'acquérir une connaissance plus approndie des différents paysages et écosystèmes de Charlevoix, plusieurs centres d'interprétation de la nature sont à leur disposition ${ }^{3}$. Ainsi, le Parc des Grands-Jardins, une des aires centrales de la Réserve de la biosphère de Charlevoix, situé au nord de Saint-Urbain (route 381 ), possède un centre d'interprétation et plusieurs sentiers de randonnée. L'un de ces sentiers mène au sommet du mont du Lac des Cygnes à près de 1000 mètres et permet de découvrir lă géomorphologie de la région, ainsi que l'étagement de la végétation en altitude et la flore de taïga et de toundra des hauts sommets. Le pare régional des Hautes-Gorges-dela-rivière-Malbaie, qui est lui aussi une aire protégée de lạ Réserve de la biosphère, offre égallement plusicurs sentiers de randonnée permettant d'observer l'étagement de la végétation sur de courtes distances et les formes glaciaires remarquables qui bordent la vallée. Le Domaine récréo-touristique *Les Palissades*, au nord de Saint-Siméon (route 170) com- porte un réseau de sentiers pédestres et des activités favorisant l'observation et l'interprétation des écosystèmes forestiers. Le Centre écologique de Port-au-Saumon organise des activités éducatives concernant l'environnement de la région. Le Centre d'interprétation et d'observation de Pointe-Noire, à Baie-Sainte-Catherine, a pour mission de faire découvír les richesses du parc marin Saguenay-SaintLaurent. De plus, un organisme privé de Baie-Saint=Paul, Randonnés NatureCharlevoix, organise des excursions d'interprétation de l'astroblème de Charlevoix ainsi que plusieurs autres excursions permettant la découverte et la connaissance des divers aspects de la nature de Charlevoix.

\section{CONCLUSION}

La succession de vallées, de plateaux. d'escarpements et de hauts sommets sur des distances relativement courtes en bordure de l'estuaire du Saint-Laurent détermine une variété de formes topographiques et d'écosystèmes possédant des qualités pittoresques saisissantes et qui constituent l'un des attraits majeurs des paysages de Charlevoix. Dans 1'Est du Canada, la possibilité de découvrir, sur de faibles distances, des formes allant des montagnes à un estuaire marin ne se retrouve guère qu'en Gaspésie et dans certaines parties de l'île de Terre-Neuve. La spécificité des paysages de Charlevoix tient à l'originalité et à l'ampleur des évênements géologiques qui ont faconné la région, ainsi qu'aux phếnomènes de contact et d'étagement qui marquent les composantes de ces paysages. Contacts géologiques entre les roches du Bouclier précambrien, des basses-terres du SaintLaurent et des Appalaches. Contact de la mer et de la montagne engendrant des contrastes climatiques spécifiques. Contact en latitude et en altitude entre les zones végétales de la forêt feuillue et de la forêt coniférienne, puis de végétation alpine des hauts sommets. Contact des paysages agricoles et forestiers. Contact des eaux douces et salées dans le moyen estuaire du Saint-Laurent et des eaux de tempérạture et salinité différentes à l'embouchure du Saguenay, etc. Enfin, on ne doit pas oublier l'empreinte de l'occupation humaine qui a modilié ces paysages à divers degrés et a contribué dans certains cas à leur harmonie ou à leur dégradation. La diversité et l'originalité des milieux qui caractérisent les paysages de Charlevoix justifient done pleinement le statut de Réserve mondiale de la biosphère qui a 
ếté octroyé à la région par l'Unesco en 1989.

\section{NOTES}

1 II est quelque peu témếraire de vouloir prếsenter la richesse et la diversité des paysages de Charlevoix en quelques pages. Dans le texte qui suit nous avons tenté d'effectuer un résumé schématique des grands traits de la nature de Charlevoix, sans prétendre couvrir l'ensemble du sujet. Pour acquérir une connaissance plus complète des attraits naturels et humains de Charlevoix, les lecteurs sont invités à parcourir le magnifique ouvrage de synthèse et de vulgarisation de Jean Des Gagniers intitulé Charlevoix. pays enchanté (Presses de l'Université Laval, 1994).

2 L'astroblème désigne les phénomènes gé̉ologiques et les formes de relief résultant de l'impact d'une météorite à la surface de la terre (voir Rondot, 1979 et 1995).

3 Le Guide touristique, Charlevoix, préparế et diffusé par l'Association touristique régionale de Charlevoix contient d'excellents renseignements sur les points d'accès pour la découverte des attraits naturels de la région.

\section{BIBLIOGRAPHIE}

Association touristique régionale de Charlevoix et Tourisme Québec, (1997), Guide touristique. Charlevoix, Quebec, Tourisme Québec, 112 p.

Association touristique régionale de Charlevoix et col... (1988), Résene mondiale de la biosphère de Charlevoix : formulaire de demande, ATR de Charlevoix, $152 \mathrm{p}$.

Des Gagniers, Jean, (1994), Charlevoix, pays enchanté, Québec, Les Presses de l'Universilé Laval, $445 \mathrm{p}$.

Groupe Paisage, (1976) Inventaire et analyse des sites el arrondissemenis naurels de Charlevoix. Les morphologies, Québec, Université Laval, Département de géographie, 139 p.

Raveneau. Jean. (1977), «Analyse morphologique, classification et protection des paysages: le cas de Charlevoixm, Cahiers de géographie du Québec, vol, 21, nos 53-54, p. 135-186.

Rondot, Jehan, (1979), Asiroblème de Charlevoix, anorthosite de Saint-Urbain et stratigraphie, Sté-Foy, Université Laval, Dép. de géologic, Faculté des sciences et génie, $38 \mathrm{p}$.

Rondot, Jehan, (1995), Les impacts météorïtiques: à l'exemple de ceux du Québec, Beauport, Qué.., les Publications MNH inc, 157 p.

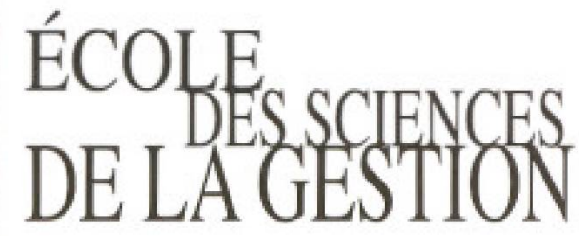

\author{
MAÎTRISE \\ EN GESTION \\ ET
}

PLANIFICATION DU TOURISME

POUR

OBJECTIF : FORMER DES GESTIONNAIRES DE HAUT NIVEAU ET DES SPÉCIALISTES

DE LA PLANIFICATION STRATÉGIQUE

DANS LE SECTEUR DU TOURISME.

PROGRAMME DE 45 CRÉDITS,

À TEMPS PLEIN OU À TEMPS PARTIEL

\section{EXIGENCES :}

- Diplôme universitaire de premier cycle

- Expérience de travail de deux ans

OU, exceptionnellement

- Expérience d'au moins cinq ans à titre de professionnel dans une entreprise touristique

\section{RENSEIGNEMENTS:}

École des sciences de la gestion de l'UOAM Département d'études urbaines et touristiques Maîtrise en gestion et planification du tourisme C.P. 8888, succ.Centre-Ville Montrëal (Québec) H3C 3P8

Téléphone: (514) 987-3000, poste 4750 Télécopieur.: (514) 987-7827

\section{UQĀMESG}

RÉPONDRE

AUX

BESOINS

D'UNE

INDUSTRIE

EN PLEIN

ESSOR. 